\title{
钴催化末端炔烃符合马氏规则的硼氢化反应
}

\author{
袁丹姚英明*
}

(苏州大学材料与化学化工学部 江苏苏州 215123)

\section{Cobalt-Catalyzed Markovnikov-Type Selective Hydroboration of Terminal Alkynes}

\author{
Yuan, Dan Yao, Yingming* \\ (College of Chemistry, Chemical Engineering and Materials Science, Soochow University, Suzhou, Jiangsu 215123)
}

不饱和化合物的嗍氢化反应，即烯烃、炔烃、醛、 酮等与嗍烷的加成反应，是一个具有 $100 \%$ 原子经济性 的反应，可用于制备一系列含嗍的化合物，这些含硼试 剂在合成化学中具有重要的应用, 比如用于 SuzukiMiyaura 偶联反应、Petasis 反应等, 可以方便地构建药 物化学、材料化学等领域的一些重要化合物. 因此, 该 反应近年来得到了越来越多的关注 ${ }^{[1]}$.

嗍氢化反应存在化学选择性和区域选择性的问题. 以炔烃和硼烷的反应为例 (Scheme 1), 它可以发生一次 加成反应, 转化为烯基嗍酸酯; 也可以发生两次加成反 应生成烷基二硼酸酯. 同时, 该加成反应既可以得到符 合马氏规则的产物，也可以得到反马氏规则的产物.

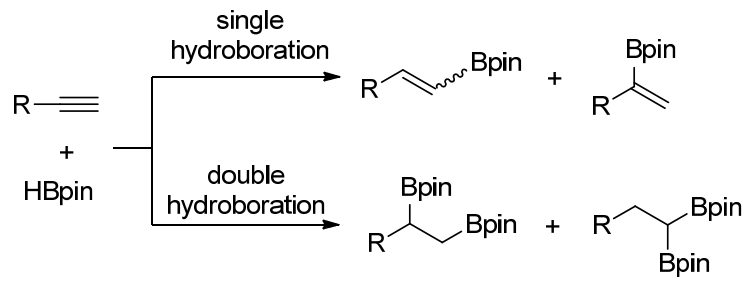

图式 1 炔烃和片哪醇嗍烷的嗍氢化反应

Scheme 1 Hydroboration of alkynes with Hbpin

炔烃和嗍烷的选择性一次加成反应是制备烯基硼 酸酯的重要方法, 反应一般主要得到反马氏规则的加成 产物 ${ }^{[2]}$, 而得到马氏规则加成产物的报道则很少 ${ }^{[3]}$. 已 有的报道主要集中在利用铜、钯等配合物催化该反应， 反应中间体为含有金属-硼键的化合物. 但这类反应体 系存在一定的局限性, 比如所用的含嗍化合物一般为双 嗍试剂, 往往会产生当量的废弃物等 ${ }^{[3]}$. 利用金属氢化
物催化炔烃与单嗍烷的硼氢化反应仍然面临很大的挑 战.

最近, 浙江大学化学系陆展团队开发了金属钴催化 末端炔烃与单硼烷的硼氢化反应，得到符合马氏规则的 产物 ${ }^{[4]}$. 他们利用噁唑环修饰 2-喹啉羧酰胺骨架, 得到 配体 $N$-(噁唑啉基苯基)喹啉-2-羧酰胺( $\mathrm{OPQC}$ ), $\mathrm{OPQC}$ 与 $\mathrm{Co}(\mathrm{OAc})_{2}$ 一起可高选择性地催化末端炔烃的硼氢化反 应，得到 $\alpha$-烯基硼酸酯(Scheme 2). 该方法的化学选择 性和区域选择性都很高, 且对官能团有很好的兼容性, 可以实现带有炔基的(保护的)醇、醚、硫醚、卤代烃、 腈、酯等 27 种化合物向烯基硼酸酯的转化.

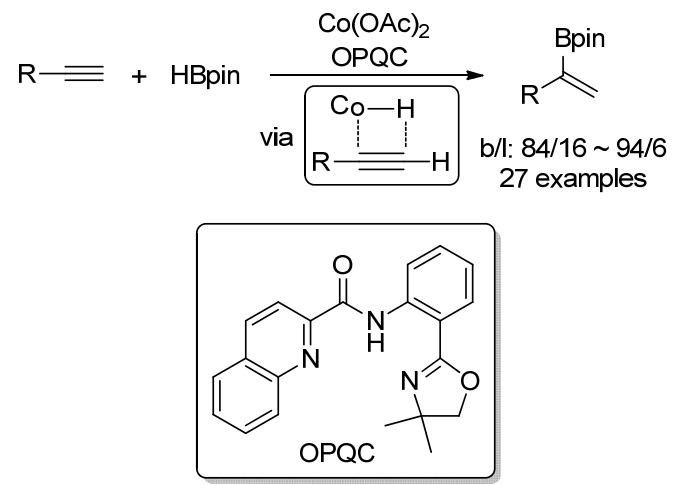

图式 $2 \mathrm{Co}(\mathrm{OAc})_{2}$ 和 $\mathrm{OPQC}$ 催化的炔烃和频哪醇嗍烷的硼氢 化反应

Scheme 2 Hydroboration of alkynes with HBpin catalyzed by $\mathrm{Co}(\mathrm{OAc})_{2} / \mathrm{OPQC}$

值得注意的是，一些具有生物活性且带有末端㶧基 的底物也可以转化为相应的烯基硼酸酯(3ab), 为这些 复杂化合物的后修饰提供了方便有效的方法.

* Corresponding author. E-mail: yaoym@suda.edu.cn. Published online February 19, 2021. 


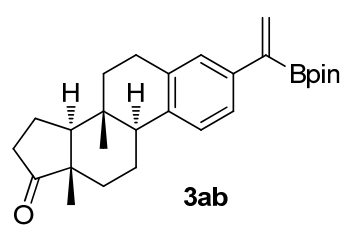

利用这种方法, 作者进行了克级产物的合成, 并且 实现了其后续的转化, 比如将烯基硼酸酯转化为烯基叠 氮(5)、二芳基烯烃(6)、环状三聚体(7)等.<smiles>C=C(N)c1ccc(Br)cc1</smiles><smiles>C=C(c1ccc(Br)cc1)c1ccc(C(=O)OC)cc1</smiles><smiles></smiles>

机理研究表明, 反应的活性中间体可能是配体 OPQC 稳定的钴氢化物 A (Scheme 3). 其催化循环过程 为炔烃与 $\mathbf{A}$ 中的钴配位, 随后发生末端炔烃插入 $\mathrm{Co}-\mathrm{H}$ 键, 生成 $\alpha$ 烯基钴化合物 C. C 与 HBpin 发生 $\sigma$-键复分 解反应生成烯基硼酸酯, 并再生钴氢化物 A. 进一步氞 代实验证明, 生成的烯基硼酸酯可以插入 $\mathbf{A}$ 中的 $\mathrm{Co}-\mathrm{H}$ 键, 生成化合物 $\mathbf{D}, \mathbf{D}$ 和目标产物相互转化.

该方法利用简单易得的催化体系, 实现了末端炔烃 和单嗍烷的选择性加成反应, 得到符合马氏规则的嗍氢 化产物, 为 $\alpha$-烯基硼酸酯的合成提供了简便且高效的方
法.

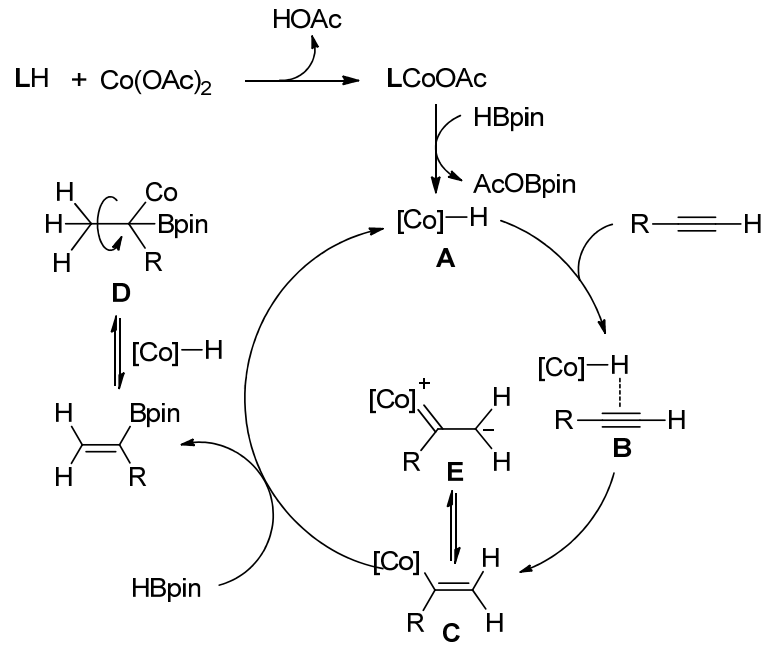

图式 3 可能的反应机理

Scheme 3 Proposed mechanism

\section{References}

[1] Wang, X.; Wang, Y.; Huang, W.; Xia, C.; Wu, L. ACS Catal. 2021, 11,1 .

[2] Selected reports: (a) Wang, Y.; Guan, R.; Sivaguru, R.; Cong, X.; Bi, X. Org. Lett. 2019, 21, 4035.

(b) Zhang, G.; Li, S.; Wu, J.; Zeng, H.; Mo, Z.; Davis, K.; Zheng, S. Org. Chem. Front. 2019, 6, 3228.

(c) Gorgas, N.; Alves, L. G.; Stöger, B.; Martins, A. M.; Veiros, L. F.; Kirchner, K. J. Am. Chem. Soc. 2017, 139, 8130 .

[3] (a) Jang, H.; Zhugralin, A. R.; Lee, Y.; Hoveyda, A. H. J. Am. Chem. Soc. 2011, 133, 7859 .

(b) Yoshida, H.; Takemoto, Y.; Takaki, K. Chem. Commun. 2014, $50,8299$.

(c) Ojha, D. P.; Prabhu, K. R. Org. Lett. 2016, 18, 432.

[4] Chen, J.; Shen, X.; Lu, Z. Angew. Chem., Int. Ed. 2021, 60, 690. 